\title{
THE
}

\section{A General Strategy to Make an On-Demand Library of Structurally and Functionally Diverse SERS Substrates}

Buddini Iroshika Karawdeniya

University of Rhode Island

Y.M. Nuwan D.Y. Bandara

University of Rhode Island

Julie C. Whelan

University of Rhode Island

Jason R. Dwyer

University of Rhode Island, jason_dwyer@uri.edu

Follow this and additional works at: https://digitalcommons.uri.edu/chm_facpubs

The University of Rhode Island Faculty have made this article openly available.

Please let us know how Open Access to this research benefits you.

This is a pre-publication author manuscript of the final, published article.

Terms of Use

This article is made available under the terms and conditions applicable towards Open Access

Policy Articles, as set forth in our Terms of Use.

\section{Citation/Publisher Attribution}

Karawdeniya, B. I, Bandara, Y.M. N. D.Y., Whelan, J. C., \& Dwyer, J. R. (2018). A General Strategy to Make an On-Demand Library of Structurally and Functionally Diverse SERS Substrates. ACS Appl. Nano Mater, 1(2), 960-968. doi: 10.1021/acsanm.7b00385

Available at: http://dx.doi.org/10.1021/acsanm.7b00385

This Article is brought to you for free and open access by the Chemistry at DigitalCommons@URI. It has been accepted for inclusion in Chemistry Faculty Publications by an authorized administrator of DigitalCommons@URI. For more information, please contact digitalcommons-group@uri.edu. 


\section{A General Strategy to Make an On-Demand Library of Structurally and Functionally Diverse SERS}

\section{Substrates.}

Buddini Iroshika Karawdeniya, Y. M. Nuwan D. Y. Bandara, Julie C. Whelan, and Jason R. Dwyer*.

Final version of manuscript available at ACS Appl. Nano Mater., 2018, 1 (2), pp 960-968

DOI: $10.1021 /$ acsanm.7b00385 (Supporting information available for free at https://pubs.acs.org/doi/suppl/10.1021/acsanm.7b00385) 


\section{A General Strategy to Make an On-Demand Library of Structurally and Functionally Diverse SERS}

\section{Substrates.}

Buddini Iroshika Karawdeniya, Y. M. Nuwan D. Y. Bandara, Julie C. Whelan, and Jason R. Dwyer*

Department of Chemistry, University of Rhode Island, 140 Flagg Road, Kingston, RI, 02881, United States.

Surface enhanced Raman spectroscopy; SERS; electroless plating; metallization.

ABSTRACT Surface-enhanced Raman spectroscopy (SERS) is a powerful technique for sensing molecules proximal to suitable coinage metal surfaces. The physical structure of the SERS-active metal layer and its support is a key design parameter inspiring considerable, and frequently specialized, efforts in substrate fabrication. The necessary gold film structure can arise from both the metallization process and the underlying support structure, and the structure of the support can deliver additional functions including analytical capabilities such as physical filtering. We used electroless plating as a general approach to create a library of SERS substrates: SERSactive gold films on a range of supports made from a variety of materials, made with a mixture of simple and complex fabrication histories, and offering a selection of structurally-derived functions. The result was that supports with existing functions had their capabilities enhanced by the addition of SERS sensing. Electroless plating thus offers a host of beneficial characteristics 
for nanofabricating multifunctional SERS substrates, including: tolerance to substrate composition and form factor; low equipment overhead requirements; process chemistry flexibility - including compatibility with conventional top-down nanofabrication; and a lengthy history of commercial application as a simple metallization technique. We gold-plated standard nanofabrication-compatible silicon nitride support surfaces with planar and porous architectures, and with native and polymer-grafted surface chemistries. We used the same plating chemistry to form SERS-active gold films on cellulose fibers arrayed in commercial filter paper and formed into nanocellulose paper. In a functional sense, we used electroless plating to augment nanoporous filters, chromatography platforms, and nanofabrication building blocks with SERS capability.

Introduction. Surface-enhanced Raman spectroscopy (SERS) is a tool at the forefront of chemical analysis for analytes ranging from single molecules to bacterial cells. ${ }^{1-5}$ Raman enhancement is engineered by tuning SERS substrate design parameters such as elemental composition; the size and shape of nanoscale elements; close-range interparticle spacing responsible for hot spots; and patterning of solid substrates that can include ordered and random hierarchies across short, long, and multiple length scales., 3, 6-10 Physical structure of the SERSactive metal layer - either its inherent structure or the structure imposed upon it by an underlying support layer-is a critical and performance-determining factor. Considerable effort has been devoted to crafting a host of solid-supported SERS substrates, with results that inspire further efforts to improve and expand fabrication options, sensing capabilities, and sensing performance. 1, 3, 7-26 Top-down nanofabrication using conventional and unorthodox approaches 
can produce exquisitely structured substrates, but can require substantial practitioner expertise along with expensive, specialized, and complicated instrumentation, and can moreover substantially limit the palette of fabrication materials. SERS substrates developed outside the material and processing constraints of conventional micro- and nanofabrication have been compelling. Both approaches and material sets hold promise. We sought, therefore, to develop a general route for nanofabricating SERS substrates that would bridge both paradigms - to draw on the strengths of each, and to be useful for both. Conventional micro- and nanofabrication approaches offer well-established, highly optimized, large-scale manufacturing capabilities for reproducibly fabricating nanoscale structures. A less conventional fabrication material such as paper offers a myriad of advantages that have driven its adoption as a material of choice for lowcost diagnostics for use in resource-limited settings. ${ }^{23,27-28}$ The genesis for the present work was the discovery that gold films we had electrolessly plated onto silicon nitride as part of a nanofabrication effort were also capable, easily and without optimization, of generating reproducible SER spectra. ${ }^{29}$ We wanted to take a variety of interesting and functional support materials and structures, and determine if a simple electroless plating process could make them SERS-active - thereby augmenting their core functions by creating multifunctional SERS substrates. This goal of multifunction does not exclude the conventional quest for maximum signal enhancement, but does require that SERS substrate evaluation be application-context dependent. Paper, for example, can support a SERS-active metal component, offers obvious advantages such as low-cost and ubiquity, and has a pore structure that could improve sensing selectivity through separations by chromatography or by physical filtering. ${ }^{18-21,23-26,28,30-42}$

Electroless plating is a robust technique for surface metallization, well-established in commercial manufacturing applications for forming decorative, electrical, and optical elements, 
and with excellent substrate tolerance. ${ }^{17,24,29,33,41,43-52}$ Objects are immersed in liquid baths, with solution access and homogeneity dictating the uniformity of the plating: rough and largearea surfaces can be coated without the geometric-including line-of-sight — constraints of physical vapor deposition. Equipment overhead is minimal, the surface being plated need not be conductive - allowing for support material tolerance - and the plating occurs without the need for external electrical power. Electroless plating is inherently different than the capture, by nonspecific or specific attachment protocols, of pre-formed, frequently ligand-coated solutionphase nanoparticles onto a surface: ${ }^{11-12,15-16,18,30-32,36-38,40}$ the electrolessly plated metal film structure, properties, and composition can be controlled through surface pretreatment, plating bath formulation, and process conditions, and can occur on a timescale that can be measured in minutes. Vitally important for our pursuit of a library of multifunctional SERS substrates, electroless plating is, in principle, compatible with coating sophisticated top-down nanofabricated, and low-cost bottom-up assembled structures and surfaces.

The term "electroless deposition" is used to describe a number of different plating mechanisms, including autocatalytic, substrate-catalyzed, and galvanic-displacement processes. $^{50}$ We adopted a single electroless plating process that had been optimized for coating nonconductive porous plastic membranes. ${ }^{49}$ In brief, a $\mathrm{Sn}$ (II) solution is used to sensitize the surface which, when treated with an ammoniacal silver nitrate solution, undergoes a redox reaction to produce a nanoscopic metallic silver layer. Gold plating is then accomplished by immersing this surface in a $\mathrm{Au}(\mathrm{I})$-containing plating bath: the aurous ions galvanically displace silver, giving gold particles that catalyze the reduction of aurous ions by formaldehyde also present in the bath. Tin-based sensitizers provide fairly indiscriminate surface sensitization, which is beneficial since tolerance to surface composition is a desired goal of our SERS substrate 
fabrication explorations. There is also much flexibility in plating chemistry after sensitization, allowing full access to the metals typically used for SERS. While silver coatings can be produced through electroless plating, the chemical stability of gold motivates our testing of gold-coated substrates for SERS activity. The use of a conventional electroless plating protocol, with only minor material-specific modifications in washing steps, allowed us to focus on support material composition and physical structure-and thereby, function - in our exploration of whether electroless plating could be a general tool for incorporating SERS sensing capabilities into already functional and structured materials and platforms.

We selected a range of support structures and material compositions to explore the generality of using electroless plating to form a library of SERS substrates. Silicon-rich LPCVD silicon nitride ( $\mathrm{SiNx}$ ) films on silicon were chosen for their ability to support a variety of nanofabricated structures and roles. ${ }^{53-55}$ Polished SiNx films ensured the nanoscale gold grain structure would be the dominant substrate structural feature. Silicon nitride films with nanoscale through-channels introduced key structural features (the individual nanochannels and the nanochannel array) underpinning designer filters and multifunctional chemical analysis platforms using plasmonic nanopores. ${ }^{56-57}$ Surface-grafting of an acrylate-based polymer generated a more subtle structural modification of the planar SiNx thin film, and was intended to increase the number of possible sensitizer interaction sites on the film. Our next selection was standard filter paper, a frequent actor in paper-based low-cost diagnostics. ${ }^{23,27}$ We explored the effect of fiber dimensions and spacing, by electrolessly plating and attempting to record SER spectra from standard filter paper and nanocellulose fiber paper - the fourth and fifth choices of material and structure. We characterized a commercial substrate (Silmeco) based on a gold-coated nanopillar array architecture $^{9}$ and etched away its gold coating to expose the sixth surface for examining 
electroless plating for SERS: a nanopillar array. Given the vastly different SERS substrate configurations, and the often severe approximations necessary to calculate enhancement factors, ${ }^{46}$ we used a comparison framework designed to compare SERS performance across disparate substrates. The method yields a SERS enhancement value (SEV), which is defined as the ratio of the analyte concentrations that produce the same instrument response by normal Raman and SER measurements. ${ }^{58}$ While spectral acquisition was formalized to allow comparisons between substrates, it nevertheless cannot account for the performance benefits of matching substrate function to a particular application.

Experimental. A detailed listing of materials and exposition of methods is provided in the Supporting Information. All substrates were electrolessly gold-plated by sequential immersion in the same series of tin (II) chloride-, ammoniacal silver nitrate-, and sodium gold sulfitecontaining solutions (Scheme S1), with appropriate rinsing steps in between immersions. The solutions were prepared as previously reported. ${ }^{29,}{ }^{59}$ Immediately prior to direct plating of bare silicon or silicon nitride surfaces, they were oxygen-plasma-treated and then etched with dilute hydrofluoric acid. The severe chemical hazards presented by hydrofluoric acid require special precautions such as those detailed in the Supporting Information. A subset of cleaned and etched planar silicon nitride supports was polymer-coated by formation of a covalently-linked sodium polyacrylate film before electroless plating, and once polymer-coated, was treated neither with plasma nor hydrofluoric acid. Silmeco gold-coated nanopillar SERS substrates were used, assupplied, for comparison measurements. These silicon nanopillar substrates were also immersed in iodide-based gold etchant and then, after plasma treatment and HF etching, electrolessly goldplated. Whatman 1 filter paper was plated without modification. Nanocellulose fibers were formed between two glass slides into a crude paper-like mat $\sim 1 \mathrm{~mm}$ thick (referred to as 
"nanocellulose paper") before plating. Surface characterization of the plated metal films was performed by field emission scanning electron microscopy (FE-SEM), x-ray photoelectron spectroscopy (XPS), and surface enhanced Raman spectroscopy (SERS).

SER spectra were acquired at an excitation wavelength of $785 \mathrm{~nm}$, with a $\sim 100 \mu \mathrm{m}$ diameter (full-width-half-maximum) beam, and at an excitation power of $\backsim 57 \mathrm{~mW}$ for cellulose and asprovided Silmeco, and $\sim 250 \mathrm{~mW}$ for all other substrates. Standard solutions of 4nitrobenzenethiol (NBT) in ethanol were prepared, covering a concentration range from $5 \times 10^{-9}$ to $1 \times 10^{-4} \mathrm{M}$. All measurements (save for replated Silmeco) were performed with the substrates immersed in the standard solutions. Substrates were immersed in standard NBT solutions and SERS spectra were recorded every 2 minutes until saturation of the signal level. Following piecewise linear background subtraction (details provided in the SI), the data was analyzed according to a framework using receiver operating characteristic (ROC) curves and kinetic analysis to calculate the $\mathrm{SEV} .{ }^{58}$

Results and Discussion. Figure 1a shows photographs of the complete set of materials before and after electroless gold plating: we use the term "support" to denote a material prior to gold plating, and the term "substrate" to denote a gold-plated support. All supports were successfully gold-plated by the series of baths of Scheme S1, as confirmed by visual inspection and XPS analysis (Figure S1). All plated substrates could be used to record SER spectra of 4nitrobenzenethiol (NBT). The support composition, however, placed restrictions on the experimental parameters. Lower excitation power was required to avoid signal saturation using the as-supplied Silmeco substrates, and substrate damage using the cellulose-based substrates. The higher excitation power left a through-hole in the paper substrate, as shown in Figure 1b, and a hollow in the thicker nanocellulose substrate after 10 exposures $(\sim 60 \mathrm{~s}$ each) when both 
were irradiated when dry; fume evolution was observed when immersed in ethanol. No damage was apparent when unplated paper that had been soaked in NBT was irradiated, so that the damage mechanism is reasonably ascribed to photothermal transduction by the gold film. This susceptibility of paper to burning is a noted benefit of using paper diagnostics in resource-limited settings where safe disposal options for biocontaminated devices may be limited. ${ }^{23,27}$

a)

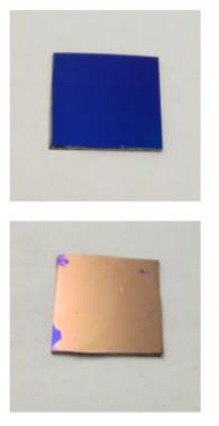

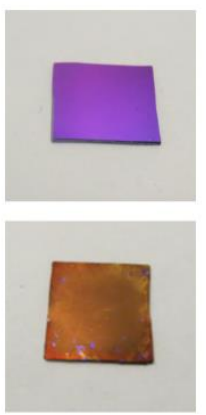

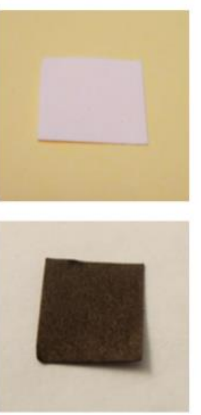

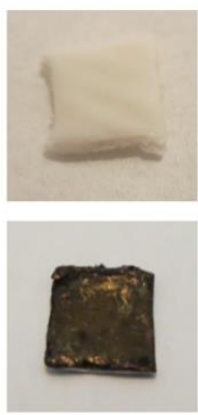

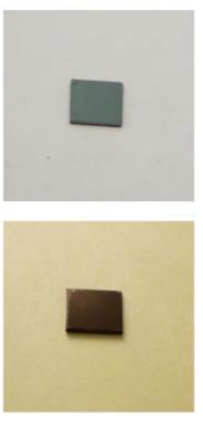

b)

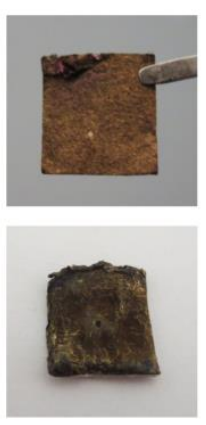

Figure 1. a) Representative substrates before (supports, top row) and after (bottom row) electroless gold plating. Left to right: Silicon nitride, polymer-grafted silicon nitride, paper, nanocellulose paper, nanopillar silicon (Silmeco etched of its as-supplied gold coating), silicon nanoporous substrates. b) Laser-induced damage at $250 \mathrm{~mW}$ sets an excitation power limit for paper (top, showing a through-hole) and nanocellulose paper (bottom, showing a hollow in the thicker substrate).

None of the (gold-free) supports produced detectable Raman spectra of NBT at a drop-cast $\sim 10^{-4} \mathrm{M}$ test dose, and the (gold-plated) substrate analyte-free background spectra were, excepting a small $\sim 1340 \mathrm{~cm}^{-1}$ peak in paper, flat and featureless in the key spectral regions used to benchmark the substrate performance (Figure S2). Figure 2 shows a representative background-subtracted SER spectrum from each substrate type using a $10^{-5} \mathrm{M}$ NBT solution. 
The principal spectral features are consistent across substrate type, including the most intense signal from the $\mathrm{NO}_{2}$ symmetric stretch, centered at $\sim 1330 \mathrm{~cm}^{-1}$ in all spectra. The intensity ratio of this peak to the $880 \mathrm{~cm}^{-1}$ ethanol peak, $R_{\mathrm{NBT} / \mathrm{EtOH}}$, was used to construct the response versus concentration curve for each substrate type in Figure S3 in the Supporting Information. These response curves had profiles typical for this class of experiment. ${ }^{58,60}$ The Raman spectral intensity at a given analyte concentration was strongly dependent upon the support material and preparation, with a substantial penalty in signal strength imposed by the excitation power limitations required by the cellulose substrates. The use of polymer-grafted silicon nitride substrates resulted in the highest signal at all concentrations compared to all other electrolessly plated substrates, most notably when compared at low analyte concentrations. To quantify the SERS performance, representative ROC curves were constructed to calculate the SEV for each substrate: $0.646 \times 10^{3}$ (paper), $0.694 \times 10^{4}$ (porous silicon nitride), $2.34 \times 10^{5}$ (nanocellulose), and $5.91 \times 10^{5}$ (silicon nitride), and at least $9.33 \times 10^{5}$ for both polymer and Silmeco substrates. Following low signal intensities in the test measurement for replated Silmeco substrates in Figure 2, we pursued structural characterization (vide infra) -instead of further spectral characterization-in an effort to understand this lower response compared to as-supplied Silmeco substrates. For the Silmeco and polymer substrates, even the measurement at the lowest concentration demonstrated a better than $90 \%$ probability of detection for a $10 \%$ probability of false alarm and due to this, we can report only a minimum SEV. ${ }^{58}$

These results emerged from proof-of-principle experiments of the general utility of electroless plating for SERS substrate creation rather than from longer-term substrate-specific optimizations. They are thus useful, when paired with the demands of a particular application, for indicating where efforts to gain additional enhancement might be warranted. The polymer- 
grafted silicon nitride is of note not simply for providing the largest SEV of our electrolessly plated substrates, but as an example of the benefits of nanoscale tailoring of SERS substrates, and for serving as a bridge between substrates based on traditional, silicon-containing nanofabrication materials, and those based on larger organic polymer fibers. More broadly, the design of a SERS substrate type should balance, in an application-specific way, the SEV and any special capabilities, such as filtering, offered by a given substrate. For example, gold films electrolessly plated onto and into these membrane filters can be used to physically optimize filter performance by tuning pore dimensions; to chemically optimize filter performance by serving as a first step in surface functionalization; and to augment filter performance by adding SERSsensing capabilities in addition to separation. ${ }^{29,}{ }^{61}$ Ultrathin, nanofabricated membrane filters, such as nanoporous silicon and silicon nitride, offer significant advantages over conventional polymer ultrafiltration membranes. ${ }^{54,}{ }^{62-70}$ Mechanically robust, unsupported ultrathin filters allow for high hydraulic and diffusive permeabilities. The material properties and ultrathin dimensions allow for the straightforward fabrication of smooth pores in controllable, welldefined sizes with narrow size distributions, and with high areal densities. The short, smooth walls do not suffer the drawbacks of flow resistance and sample losses due to the tortuosity and large surface area of conventional, thicker (polycarbonate) track-etched membranes. Such highthroughput, low-loss nanoporous membranes can be custom-fabricated with pore dimensions and characteristics optimized to filter micrometer-scale organisms such as bacteria, or even to separate macromolecules. Sensitivity might be enhanced by optimizing pore dimensions and distributions to form a nanoplasmonic array, ${ }^{56}$ but at the cost of filtration performance (and selectivity). ${ }^{57} \mathrm{~A}$ different example of the need to balance SEV and other application demands is illustrated in Figure S4: electrolessly gold-coated paper was used for the SERS readout of a 
crude paper-based assay that performed physical filtration and chromatographic separation. This multifunction capability augments the spectral selectivity of SERS for greater ease of analysis of multicomponent samples, but by no means circumscribes the utility of SERS-active paper. Indeed, the development of paper-based diagnostics has been characterized by the incorporation — by a variety of approaches, sophisticated and simple—of ever-greater function into paper-based supports. ${ }^{23,27-28,42}$

One means to create useful multifunctional SERS substrates - or even highly optimized SERSonly substrates - is through the deliberate incorporation of carefully selected structural features in the supports. The presence of pores, or voids, in a support has a number of consequences for SERS substrates: the available surface area for sensing can be diminished; the likelihood of hot spot formation can be affected, depending on the spatial extent and distribution of the voids; signal collection can be affected by scattering, line-of-sight access, and focal depth for threedimensional and structured substrates; mismatches between the excitation volume and the surfaces bearing analyte can limit reproducibility or signal magnitude; plasmonic nanopores, especially in arrays, introduce new optical considerations; and if analyte is delivered by dropcasting, the open area can profoundly affect the spatial distribution of analyte during solvent evaporation. For SERS substrates fabricated using an electroless plating step, the pores can affect the electroless deposition nucleation and growth (by imposing boundaries, for example). These factors include effects that can be much stronger than simple geometric coverage, allowing for considerable parameter space for optimizing performance through the support geometry and through the electroless plating parameters. We recorded scanning electron micrographs, with representative examples shown in Figures 3, 4, and 5, to gain preliminary structural insights, particularly with respect to the diversity of support structures that could be electrolessly plated. 
The set of micrographs showed consistently high coverage across the different replicates and substrate types.

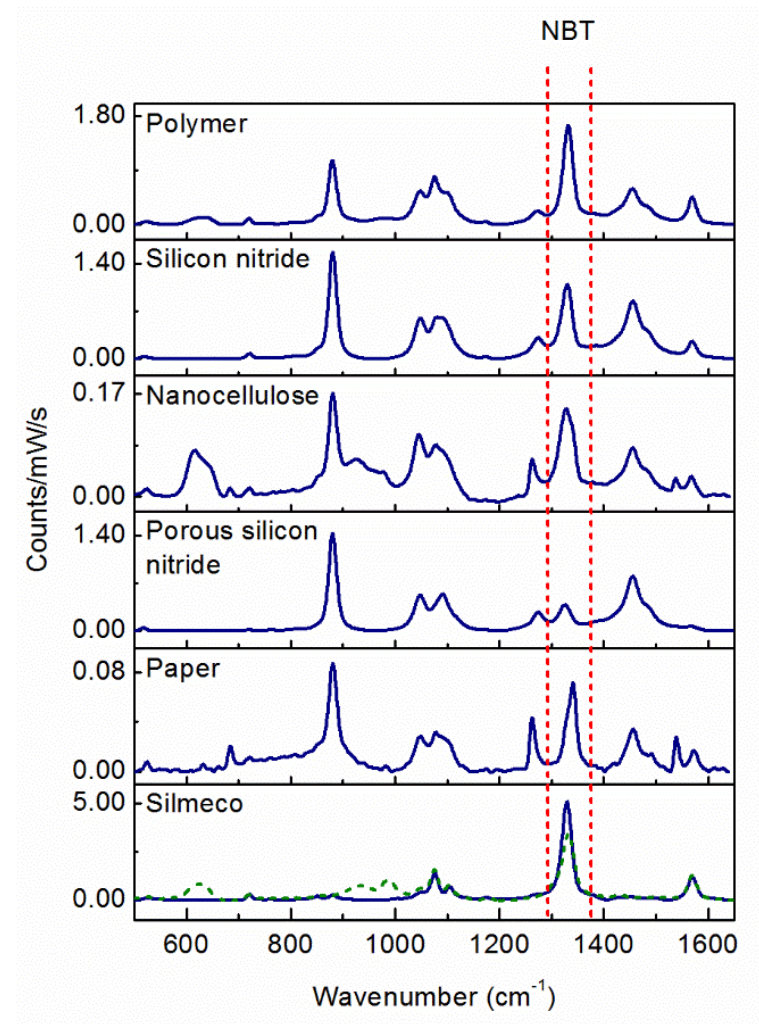

Figure 2. Representative baseline-corrected spectra of each substrate at $10^{-5} \mathrm{M}$ NBT in ethanol ( $\sim 57 \mathrm{~mW}$ for cellulose and as-supplied Silmeco; $\sim 250 \mathrm{~mW}$ for all others). The dotted spectrum in the bottom panel shows the signal (scaled $20 \times$ ) at $250 \mathrm{~mW}$ from $5 \mu \mathrm{L}$ of $1.6 \times 10^{-5} \mathrm{M}$ NBT in acetonitrile drop-cast onto the electrolessly-replated Silmeco. The vertical dotted lines denote the integration range for the NBT peak of interest.

Figure 3 provides a set of comparative micrographs of representative gold coatings on the silicon nitride-containing substrates. The uniform through-holes in the nanoporous membrane are a captivating structural feature compatible with compelling functions, ${ }^{56-57}$ and the nanoporous 
membrane was moreover free-standing between support bars (not shown) so that it was electrolessly gold-plated within the pores and on both sides of the membrane. We avoided any ultrasonic cleaning steps that might cause rupture of this thin porous membrane, and we were consistent in this purposeful omission across all substrates. The three substrates were composed of nanostructured gold films with low- and high-aspect ratio grains, but the preponderance and character of the high-aspect ratio structures differed dramatically between the substrate types. The polymer-grafted silicon nitride gold film bore the greatest number of integral high-aspect ratio features, and with a unique grain structure characterized by the prevalence of larger, sharper, and more finely substructured gold flakes that projected from the surface. These flakes provide an increase in surface area for chemisorption of the NBT, and more significantly, are nanostructured on a length scale favorable for the existence of hot spots, and with an aspect ratio amenable to signal enhancement by the lightning rod effect. ${ }^{4}$ The nanoporous substrate imposed gaps between gold grains, although on length scales optimized, in this substrate, for filtering rather than hot spot formation. ${ }^{57}$ The loss of planar substrate area might be compensated for by plating sufficiently long pores, but the nanochannel surface is normal to the conventional substrate surface, and longer pores would affect through-pore flow rates. Overall, detrimental decreases in sensitivity from surface area losses to pores may be quickly outpaced by beneficial gains to analytical performance through the selectivity and throughput that emerges from careful tuning of the pore geometry to support rapid and tuned sample filtering. 

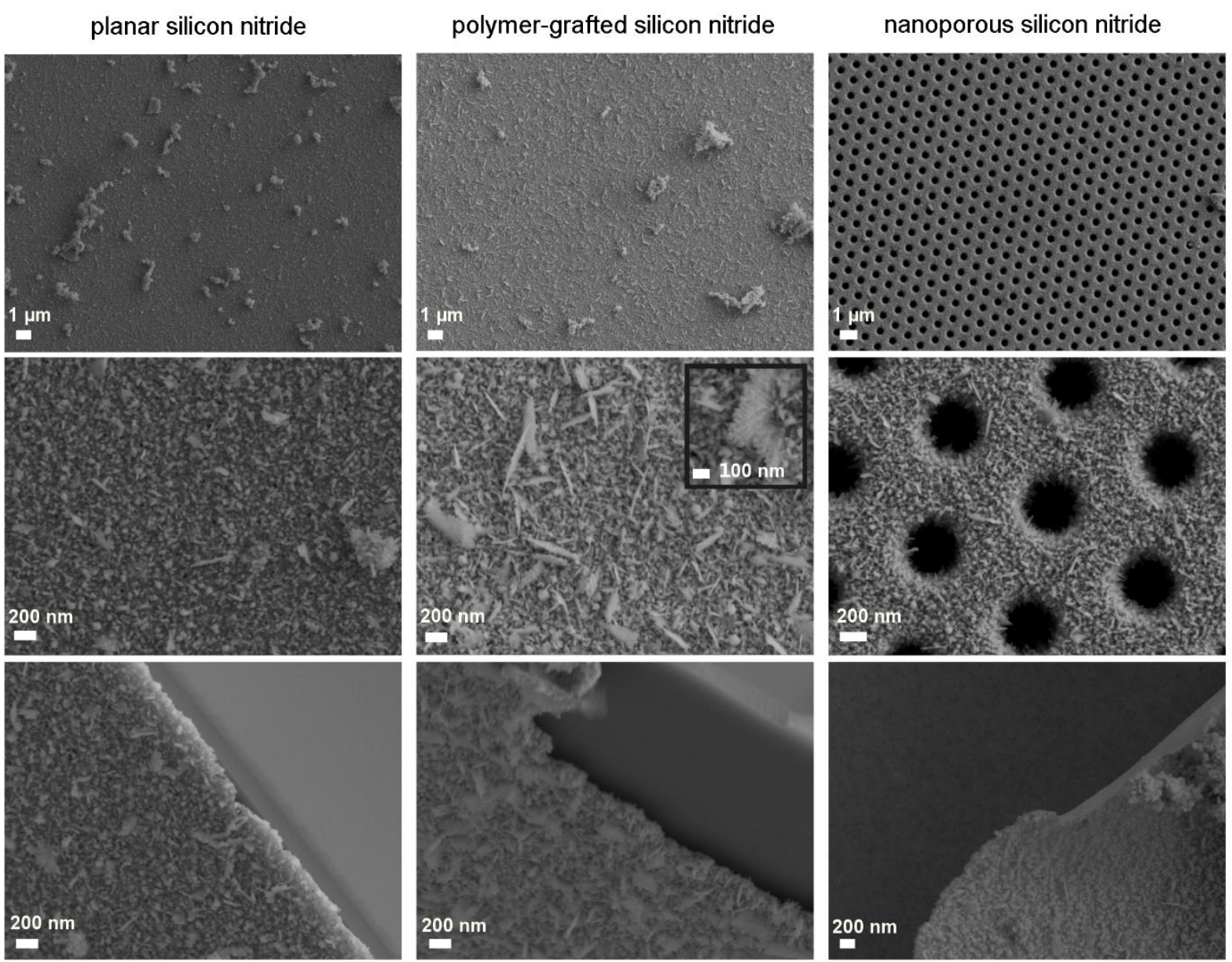

Figure 3. SEM images of, from left-to-right by column, gold-plated silicon nitride, polymergrafted silicon nitride, and nanoporous silicon nitride. The top two rows show top-down images while the bottom row shows an angled view of gold film cross-sections. The inset in the center micrograph more clearly shows a representative highly-structured flake.

Figure 4 shows scanning electron micrographs from electrolessly-plated paper and nanocellulose samples. The paper substrate was distinguished by voids between large fibers constructed of bundled nanoscale fibers. The presence of void spaces in a given layer of the paper is partially compensated by overlap with fibers in underlying layers. The pore, or void 
space, size distribution in paper can be controlled during its manufacture, and is an important metric when selecting commercial filter paper, for example. The hand-fabricated nanocellulose substrate was highly textured and convoluted, without the fiber bundling, alignment, and low packing density that produced obvious microscale voids in the paper substrate. The ability of electroless plating to coat rough, nonplanar surfaces-beyond what was seen in the plating of the curved pore walls orthogonal to the planar upper surface of the porous silicon nitride film-is dramatically illustrated by the impressive surface coverage. Thick, porous supports such as the nanocellulose paper have a large surface area for plating — distributed throughout their interiorand require a greater minimum plating solution volume than a planar support. Similarly, most of the plated gold surfaces will be able to bind analyte but will be optically inaccessible, and must be considered when aliquoting samples. Even after addressing these issues, the available signal strength using the cellulose-supported substrates was limited by the lower allowable excitation intensity. The fiber-based construction of the cellulose substrates, however, is an intriguing structural design feature that can provide additional analytical capabilities such as swab sampling and chromatographic separation. ${ }^{35,44,71}$ The cellulose substrates are evocative of other fiber-mat platforms used for SERS, ${ }^{11-12,14-22}$ with paper supports being available at scale and at low cost using well-established manufacturing methods. When the ability to filter or chromatographically separate a sample using a SERS-active porous substrate is desired in addition to SERS sensing, one must consider the effect of the pore size on each capability —and on the interplay between each capability. Pore size is tunable through support fabrication or through the plating timedependent thickness — within the limits of cost and available gold in the plating bath—of the plated gold layer. The flexibility, simplicity, and ease-of-handling of these nanofiber-based 
substrates stand in stark contrast to the more delicately engineered Silmeco nanopillar arrays, particularly for applications in resource-challenged settings.
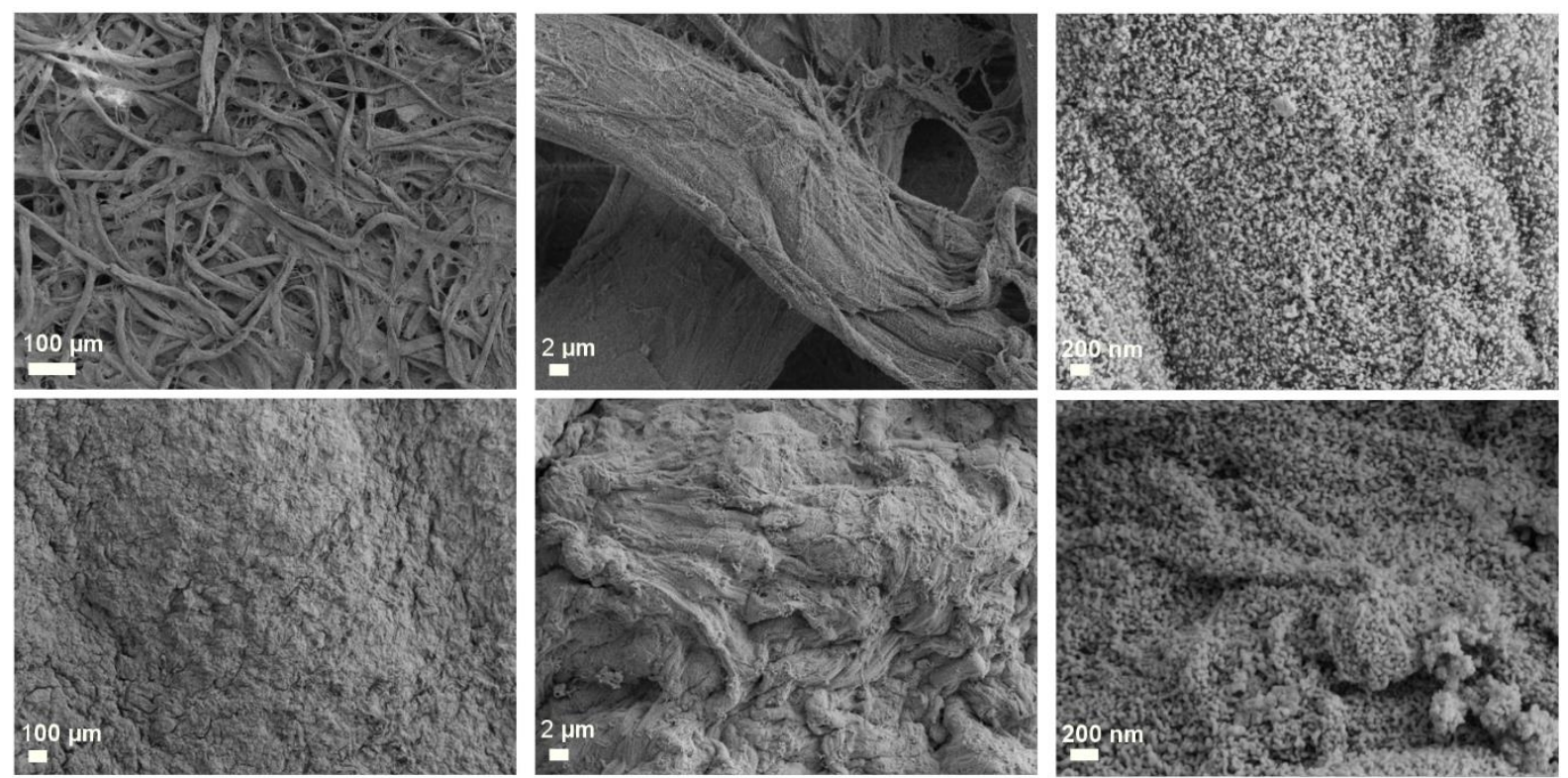

Figure 4. SEM images of gold-plated paper substrates (top row) and gold-plated nanocellulose paper substrates (bottom row).

The superb Raman enhancement that the nanopillar substrates provided when used as-supplied, without modification, reinforces the utility of rationally patterning traditional micro- and nanofabrication materials to create SERS substrates. One must, however, be careful during handling and solution processing to prevent unwanted damage or modification of such highaspect ratio features: ${ }^{9}$ the gold-etched surface shows some broken nanopillars. SEM images in Figure 5 show that our general process chemistry was able to successfully electrolessly goldplate a nanopillar array. The figure shows a section of electrolessly plated gold film that had peeled back from the nanopillar array surface: the surface of the gold film formerly in contact with the nanopillar array clearly shows dark areas that are consistent with electroless gold plating 
around extant nanopillars of the array. The dominant structural motifs of as-supplied Silmeco substrates - recognizable individual gold-encrusted nanopillars with limited numbers of contact points between nanopillars to yield likely hot spots-were not conspicuous in our top-down micrographs of the electrolessly plated substrates. This absence of a key SERS-associated (nano)structure is the most significant contributor to the dramatic loss of spectral intensity when using replated Silmeco. While several of the dark areas of the underside of the gold film are evocative of plating around nanopillars likely already leaning together ${ }^{9}$, optimization of the electroless plating for this nanopillar support would be necessary to deliver the engineered hot spots of the as-supplied substrate. The most reasonable starting point for such an optimization would be to plate pristine gold-free nanoarrays so that the distance between the gold regions of adjacent nanopillars could be controlled by the plating kinetics and time, and any post-plating drying-induced pillar leaning. Producing a nanoarray surface by etching gold from the assupplied Silmeco handicaps the subsequent replating with the initial structural modification of hot spot formation and the likely damage to the nanoarray of the gold etching step. Nevertheless, the robust gold film formed around nanopillars in this particular micrograph is a compelling reminder of the ability of electroless plating to plate nanoscale structures, and its ability to create, without substantial equipment overhead, SERS substrates from highly engineered supports.
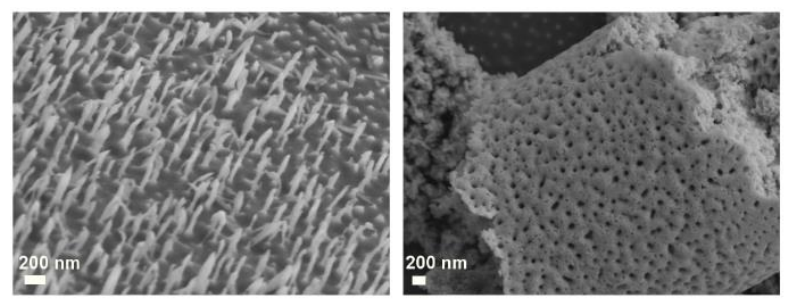

Figure 5. SEM image of a nanopillar substrate after gold etch (left), and with an electrolessly plated gold film peeled off of the underlying nanopillar support (right). 
Conclusions. Electroless plating is a robust method for fashioning a variety of materials, exhibiting a range of structural features and capabilities, into SERS-active substrates. The general electroless plating procedure we employed was able to successfully plate gold onto planar, porous, nanopillar, and fibrous surfaces; into well-defined nanochannels and variablysized void volumes; onto traditional nanofabrication-compatible materials; and onto less conventional device platform materials such as paper that are important in the domain of lowcost diagnostics. All resulting substrates in our library were capable of generating SER spectra. This electroless plating approach produced nanostructured films where the size, shape, and position of the gold grains could be tuned by the particular material and form factor of the support material being plated, and this tuneability was evident from both microscopic imaging and SERS intensities. The underlying support structure for the gold plating did more than imprint structure on the gold film, though. Electroless plating of already functional structured supports created multifunctional SERS substrates. The force of the work presented here is thus both foundational and prospective: there is much promise in exploring electroless plating-including extensions such as patterned electroless plating ${ }^{51,55}$ — as a straightforward, robust, and lowoverhead method to create custom SERS-active substrates that augment the compelling material properties, structures, and capabilities of their supports. Multifunctional SERS substrates require a rich, and application-specific, context and framework for design and performance evaluation. The substrate must, of course, generate a useful Raman spectrum, but the particular implementation - from design and fabrication to end-use - dictates the balance between Raman enhancement and other capabilities such as integral sample processing. This balance dictates how to tune the electroless plating process chemistry, and the support structure, to optimize the 
SERS substrate. We believe that electroless plating has great potential in the creation of multifunctional SERS substrates useful for answering a host of design and sensing challenges.

\section{ASSOCIATED CONTENT}

\section{Supporting Information.}

The following files are available free of charge.

Experimental details, methods and sample characterizations (PDF)

\section{AUTHOR INFORMATION}

\section{Corresponding Author}

*jason_dwyer@uri.edu

\section{Author Contributions}

The manuscript was written through contributions of all authors. All authors have given approval to the final version of the manuscript.

\section{Funding Sources}

This research has been supported by NSF CAREER award CBET-1150085, by NSF EPSCoR Cooperative Agreement \#IIA-1330406, and by the University of Rhode Island including URI graduate fellowships for Y. M. Nuwan D. Y. Bandara and Buddini Iroshika Karawdeniya.

\section{ACKNOWLEDGMENT}


B. L. Lucht research group at the University of Rhode Island for use of their XPS system, and for assistance in XPS data acquisition. This research has been supported by NSF CAREER award CBET-1150085, by NSF EPSCoR Cooperative Agreement \#IIA-1330406, and by the University of Rhode Island including URI graduate fellowships for YMNDYB and BIK.

\section{ABBREVIATIONS}

SERS, Surface enhanced Raman spectroscopy; LPCVD, low-pressure chemical vapor deposition; $\mathrm{SiN}_{\mathrm{x}}$, (silicon-rich) LPCVD silicon nitride; SEV, SERS enhancement value; FESEM, field emission scanning electron microscopy; XPS, x-ray photoelectron spectroscopy; ROC, receiver operating characteristic; NBT, 4-nitrobenzenethiol;

\section{REFERENCES}

1. Yang, L.; Yan, B.; Premasiri, W. R.; Ziegler, L. D.; Negro, L. D.; Reinhard, B. M., Engineering Nanoparticle Cluster Arrays for Bacterial Biosensing: The Role of the Building Block in Multiscale SERS Substrates. Adv. Funct. Mater. 2010, 20, 2619-2628.

2. Patel, I. S.; Premasiri, W. R.; Moir, D. T.; Ziegler, L. D., Barcoding Bacterial Cells: A SERS-Based Methodology for Pathogen Identification. Journal of Raman Spectroscopy 2008, $39,1660-1672$.

3. Stiles, P. L.; Dieringer, J. A.; Shah, N. C.; Van Duyne, R. P., Surface-Enhanced Raman Spectroscopy. Annu. Rev. Anal. Chem. 2008, 1, 601-626.

4. Aroca, R., Surface-Enhanced Vibrational Spectroscopy. John Wiley \& Sons Ltd: Chichester, West Sussex PO19 8SQ, England, 2006. 
5. Surface Enhanced Raman Spectroscopy: Analytical, Biophysical and Life Science Applications. Wiley-VCH Verlag GmbH \& Co. KGaA: 2010; p I-XXII, 1-331.

6. Murphy, C. J.; Sau, T. K.; Gole, A. M.; Orendorff, C. J.; Gao, J.; Gou, L.; Hunyadi, S. E.; Li, T., Anisotropic Metal Nanoparticles: Synthesis, Assembly, and Optical Applications. J. Phys. Chem. B 2005, 109, 13857-13870.

7. Perney, N. M. B.; Baumberg, J. J.; Zoorob, M. E.; Charlton, M. D. B.; Mahnkopf, S.; Netti, C. M., Tuning Localized Plasmons in Nanostructured Substrates for Surface-Enhanced Raman Scattering. Opt. Express 2006, 14, 847-857.

8. Tabatabaei, M.; Najiminaini, M.; Davieau, K.; Kaminska, B.; Singh, M. R.; Carson, J. J. L.; Lagugné-Labarthet, F., Tunable 3d Plasmonic Cavity Nanosensors for Surface-Enhanced Raman Spectroscopy with Sub-Femtomolar Limit of Detection. ACS Photonics 2015, 2, 752 759.

9. Schmidt, M. S.; Hübner, J.; Boisen, A., Large Area Fabrication of Leaning Silicon Nanopillars for Surface Enhanced Raman Spectroscopy. Adv. Mater. 2012, 24, OP11-OP18.

10. Gopinath, A.; Boriskina, S. V.; Reinhard, B. M.; Dal Negro, L., Deterministic Aperiodic Arrays of Metal Nanoparticles for Surface-Enhanced Raman Scattering (SERS). Opt. Express 2009, 17, 3741-3753.

11. Aldeanueva-Potel, P.; Correa-Duarte, M. A.; Alvarez-Puebla, R. A.; Liz-Marzán, L. M., Free-Standing Carbon Nanotube Films as Optical Accumulators for Multiplex Serrs Attomolar Detection. ACS Appl. Mater. Interfaces 2010, 2, 19-22. 
12. He, D.; Hu, B.; Yao, Q.-F.; Wang, K.; Yu, S.-H., Large-Scale Synthesis of Flexible FreeStanding SERS Substrates with High Sensitivity: Electrospun Pva Nanofibers Embedded with Controlled Alignment of Silver Nanoparticles. ACS Nano 2009, 3, 3993-4002.

13. Manas, R. G.; Zhida, X.; Elaine, B.; Hoang, N.; Jerald, A. B.; Cindy, L.; Robin, M.; Mihail, B.; Allan, S. P. C.; Tiziana, C. B.; Liu, G. L., Rigorous Surface Enhanced Raman Spectral Characterization of Large-Area High-Uniformity Silver-Coated Tapered Silica Nanopillar Arrays. Nanotechnology 2010, 21, 395701.

14. Dawson, P.; Duenas, J. A.; Boyle, M. G.; Doherty, M. D.; Bell, S. E. J.; Kern, A. M.; Martin, O. J. F.; Teh, A. S.; Teo, K. B. K.; Milne, W. I., Combined Antenna and Localized Plasmon Resonance in Raman Scattering from Random Arrays of Silver-Coated, Vertically Aligned Multiwalled Carbon Nanotubes. Nano Lett. 2011, 11, 365-371.

15. Zhang, C.-L.; Lv, K.-P.; Cong, H.-P.; Yu, S.-H., Controlled Assemblies of Gold Nanorods in Pva Nanofiber Matrix as Flexible Free-Standing SERS Substrates by Electrospinning. Small 2012, 8, 648-653.

16. Roskov, K. E.; Kozek, K. A.; Wu, W.-C.; Chhetri, R. K.; Oldenburg, A. L.; Spontak, R. J.; Tracy, J. B., Long-Range Alignment of Gold Nanorods in Electrospun Polymer Nano/Microfibers. Langmuir 2011, 27, 13965-13969.

17. Zhang, L.; Gong, X.; Bao, Y.; Zhao, Y.; Xi, M.; Jiang, C.; Fong, H., Electrospun Nanofibrous Membranes Surface-Decorated with Silver Nanoparticles as Flexible and Active/Sensitive Substrates for Surface-Enhanced Raman Scattering. Langmuir 2012, 28, 1443314440. 
18. Lee, C. H.; Hankus, M. E.; Tian, L.; Pellegrino, P. M.; Singamaneni, S., Highly Sensitive Surface Enhanced Raman Scattering Substrates Based on Filter Paper Loaded with Plasmonic Nanostructures. Anal. Chem. 2011, 83, 8953-8958.

19. Yu, W. W.; White, I. M., Inkjet Printed Surface Enhanced Raman Spectroscopy Array on Cellulose Paper. Anal. Chem. 2010, 82, 9626-9630.

20. Polavarapu, L.; Porta, A. L.; Novikov, S. M.; Coronado-Puchau, M.; Liz-Marzán, L. M., Pen-on-Paper Approach toward the Design of Universal Surface Enhanced Raman Scattering Substrates. Small 2014, 10, 3065-3071.

21. Berthod, A.; Laserna, J. J.; Winefordner, J. D., Analysis by Surface Enhanced Raman Spectroscopy on Silver Hydrosols and Silver Coated Filter Papers. J. Pharm. Biomed. Anal. 1988, 6, 599-608.

22. Qu, L.-L.; Li, D.-W.; Xue, J.-Q.; Zhai, W.-L.; Fossey, J. S.; Long, Y.-T., Batch Fabrication of Disposable Screen Printed SERS Arrays. Lab on a Chip 2012, 12, 876-881.

23. Then, W. L.; Garnier, G., Paper Diagnostics in Biomedicine. Rev. Anal. Chem 2013, 32, 269-294.

24. Penn, M. A.; Drake, D. M.; Driskell, J. D., Accelerated Surface-Enhanced Raman Spectroscopy (SERS)-Based Immunoassay on a Gold-Plated Membrane. Anal. Chem. 2013, 85, 8609-8617.

25. Polavarapu, L.; Liz-Marzan, L. M., Towards Low-Cost Flexible Substrates for Nanoplasmonic Sensing. PCCP 2013, 15, 5288-5300. 
26. Fan, M.; Zhang, Z.; Hu, J.; Cheng, F.; Wang, C.; Tang, C.; Lin, J.; Brolo, A. G.; Zhan, H., Ag Decorated Sandpaper as Flexible SERS Substrate for Direct Swabbing Sampling. Mater. Lett. 2014, 133, 57-59.

27. Nery, E. W.; Kubota, L. T., Sensing Approaches on Paper-Based Devices: A Review. Anal Bioanal Chem 2013, 405, 7573-7595.

28. Yang, Y.; Noviana, E.; Nguyen, M. P.; Geiss, B. J.; Dandy, D. S.; Henry, C. S., PaperBased Microfluidic Devices: Emerging Themes and Applications. Anal. Chem. 2017, 89, 71-91.

29. Whelan, J. C.; Karawdeniya, B. I.; Bandara, Y. M. N. D. Y.; Velleco, B. D.; Masterson, C. M.; Dwyer, J. R., Electroless Plating of Thin Gold Films Directly onto Silicon Nitride Thin Films and into Micropores. ACS Appl. Mater. Interfaces 2014, 6, 10952-10957.

30. Zheng, G.; Polavarapu, L.; Liz-Marzan, L. M.; Pastoriza-Santos, I.; Perez-Juste, J., Gold Nanoparticle-Loaded Filter Paper: A Recyclable Dip-Catalyst for Real-Time Reaction Monitoring by Surface Enhanced Raman Scattering. Chem. Commun. 2015, 51, 4572-4575.

31. Vo-Dinh, T.; Hiromoto, M. Y. K.; Begun, G. M.; Moody, R. L., Surface-Enhanced Raman Spectrometry for Trace Organic Analysis. Anal. Chem. 1984, 56, 1667-1670.

32. Tran, C. D., Subnanogram Detection of Dyes on Filter Paper by Surface-Enhanced Raman Scattering Spectrometry. Anal. Chem. 1984, 56, 824-826.

33. Laserna, J. J.; Campiglia, A. D.; Winefordner, J. D., Surface-Enhanced Raman Spectrometry on a Silver-Coated Filter Paper Substrate. Anal. Chim. Acta 1988, 208, 21-30. 
34. Laserna, J. J.; Sutherland, W. S.; Winefordner, J. D., Microspectrometric Investigation of Active Substrates for Surface Enhanced Raman Scattering. Anal. Chim. Acta 1990, 237, 439450.

35. Lee, C. H.; Tian, L.; Singamaneni, S., Paper-Based SERS Swab for Rapid Trace Detection on Real-World Surfaces. ACS Appl. Mater. Interfaces 2010, 2, 3429-3435.

36. Ngo, Y. H.; Li, D.; Simon, G. P.; Garnier, G., Gold Nanoparticle-Paper as a ThreeDimensional Surface Enhanced Raman Scattering Substrate. Langmuir 2012, 28, 8782-8790.

37. Ngo, Y. H.; Li, D.; Simon, G. P.; Garnier, G., Effect of Cationic Polyacrylamides on the Aggregation and SERS Performance of Gold Nanoparticles-Treated Paper. J. Colloid Interface Sci. 2013, 392, 237-246.

38. Zhang, L.; Li, X.; Ong, L.; Tabor, R. F.; Bowen, B. A.; Fernando, A. I.; Nilghaz, A.; Garnier, G.; Gras, S. L.; Wang, X.; Shen, W., Cellulose Nanofibre Textured SERS Substrate. Colloids and Surfaces A: Physicochemical and Engineering Aspects 2015, 468, 309-314.

39. Niu, Z.; Fang, Y., Surface-Enhanced Raman Scattering of Single-Walled Carbon Nanotubes on Silver-Coated and Gold-Coated Filter Paper. J. Colloid Interface Sci. 2006, 303, 224-228.

40. Ota, F.; Higuchi, S.; Gohshi, Y.; Furuya, K.; Ban, M.; Kyoto, M., Some Considerations of the SERS Effect of L-Phenylalanine in the near-Infrared Region Using Silver Colloid Solution. Journal of Raman Spectroscopy 1997, 28, 849-854.

41. Wigginton, K. R.; Vikesland, P. J., Gold-Coated Polycarbonate Membrane Filter for Pathogen Concentration and SERS-Based Detection. Analyst 2010, 135, 1320-1326. 
42. Zhang, K.; Zhao, J.; Xu, H.; Li, Y.; Ji, J.; Liu, B., Multifunctional Paper Strip Based on Self-Assembled Interfacial Plasmonic Nanoparticle Arrays for Sensitive SERS Detection. ACS Appl. Mater. Interfaces 2015, 7, 16767-16774.

43. Møller, P.; Nielsen, L. P., Advanced Surface Technology. Møller \& Nielsen APS: Denmark, 2013; Vol. 1, p 594.

44. Cabalín, L. M.; Laserna, J. J., Fast Spatially Resolved Surface-Enhanced Raman Spectrometry on a Silver Coated Filter Paper Using Charge-Coupled Device Detection. Anal. Chim. Acta 1995, 310, 337-345.

45. Cheng, M.-L.; Tsai, B.-C.; Yang, J., Silver Nanoparticle-Treated Filter Paper as a Highly Sensitive Surface-Enhanced Raman Scattering (SERS) Substrate for Detection of Tyrosine in Aqueous Solution. Anal. Chim. Acta 2011, 708, 89-96.

46. Norrod, K. L.; Sudnik, L. M.; Rousell, D.; Rowlen, K. L., Quantitative Comparison of Five SERS Substrates: Sensitivity and Limit of Detection. Appl. Spectrosc. 1997, 51, 994-1001.

47. Saito, Y.; Wang, J. J.; Smith, D. A.; Batchelder, D. N., A Simple Chemical Method for the Preparation of Silver Surfaces for Efficient SERS. Langmuir 2002, 18, 2959-2961.

48. Tabakman, S. M.; Chen, Z.; Casalongue, H. S.; Wang, H.; Dai, H., A New Approach to Solution-Phase Gold Seeding for SERS Substrates. Small 2011, 7, 499-505.

49. Menon, V. P.; Martin, C. R., Fabrication and Evaluation of Nanoelectrode Ensembles. Anal. Chem. 1995, 67, 1920-1928.

50. Porter, L. A.; Choi, H. C.; Ribbe, A. E.; Buriak, J. M., Controlled Electroless Deposition of Noble Metal Nanoparticle Films on Germanium Surfaces. Nano Lett. 2002, 2, 1067-1071. 
51. Zabetakis, D.; Dressick, W. J., Selective Electroless Metallization of Patterned Polymeric Films for Lithography Applications. ACS Appl. Mater. Interfaces 2009, 1, 4-25.

52. Liu, F.-M.; Green, M., Efficient SERS Substrates Made by Electroless Silver Deposition into Patterned Silicon Structures. J. Mater. Chem. 2004, 14, 1526-1532.

53. Dwyer, J. R.; Harb, M., Through a Window, Brightly: A Review of Selected Nanofabricated Thin-Film Platforms for Spectroscopy, Imaging, and Detection. Appl. Spectrosc. 2017, 71, 2051-2075.

54. Dwyer, J. R.; Bandara, Y. M. N. D. Y.; Whelan, J. C.; Karawdeniya, B. I.; Nichols, J. W., Silicon Nitride Thin Films for Nanofluidic Device Fabrication. In Nanofluidics, 2nd Edition, 2 ed.; Edel, J.; Ivanov, A.; Kim, M., Eds. Royal Society for Chemistry: 2016; Chapter 7.

55. Bandara, Y. M. N. D. Y.; Karawdeniya, B. I.; Whelan, J. C.; Ginsberg, L. D. S.; Dwyer, J. R., Solution-Based Photo-Patterned Gold Film Formation on Silicon Nitride. ACS Appl. Mater. Interfaces 2016, 8, 34964-34969.

56. Dahlin, A. B., Sensing Applications Based on Plasmonic Nanopores: The Hole Story. Analyst 2015, 140, 4748-4759.

57. Kuiper, S.; van Rijn, C. J. M.; Nijdam, W.; Elwenspoek, M. C., Development and Applications of Very High Flux Microfiltration Membranes. Journal of Membrane Science 1998, $150,1-8$

58. Guicheteau, J. A.; Farrell, M. E.; Christesen, S. D.; Fountain, A. W.; Pellegrino, P. M.; Emmons, E. D.; Tripathi, A.; Wilcox, P.; Emge, D., Surface-Enhanced Raman Scattering (SERS) Evaluation Protocol for Nanometallic Surfaces. Appl. Spectrosc. 2013, 67, 396-403. 
59. Whelan, J. C.; Karawdeniya, B. I.; Bandara, Y. M. N. D. Y.; Velleco, B. D.; Masterson, C. M.; Dwyer, J. R., Correction to Electroless Plating of Thin Gold Films Directly onto Silicon Nitride Thin Films and into Micropores. ACS Appl. Mater. Interfaces 2015, 7, 26004-26004.

60. Massarini, E.; Wästerby, P.; Landström, L.; Lejon, C.; Beck, O.; Andersson, P. O., Methodologies for Assessment of Limit of Detection and Limit of Identification Using SurfaceEnhanced Raman Spectroscopy. Sensors and Actuators B: Chemical 2015, 207, Part A, 437-446.

61. Wirtz, M.; Yu, S.; Martin, C. R., Template Synthesized Gold Nanotube Membranes for Chemical Separations and Sensing. Analyst 2002, 127, 871-879.

62. DesOrmeaux, J. P. S.; Winans, J. D.; Wayson, S. E.; Gaborski, T. R.; Khire, T. S.; Striemer, C. C.; McGrath, J. L., Nanoporous Silicon Nitride Membranes Fabricated from Porous Nanocrystalline Silicon Templates. Nanoscale 2014, 6, 10798-10805.

63. Striemer, C. C.; Gaborski, T. R.; McGrath, J. L.; Fauchet, P. M., Charge- and Size-Based Separation of Macromolecules Using Ultrathin Silicon Membranes. Nature 2007, 445, 749-753.

64. Joshua, J. M.; Robert, N. C.; Kelly, B. M.; Jon-Paul, S. D.; Christopher, C. S.; Joshua, D. W.; Thomas, R. G., Lift-Off of Large-Scale Ultrathin Nanomembranes. Journal of Micromechanics and Microengineering 2015, 25, 015011.

65. Gironès, M.; Borneman, Z.; Lammertink, R. G. H.; Wessling, M., The Role of Wetting on the Water Flux Performance of Microsieve Membranes. Journal of Membrane Science 2005, $259,55-64$. 
66. Huang, H.; Song, Z.; Wei, N.; Shi, L.; Mao, Y.; Ying, Y.; Sun, L.; Xu, Z.; Peng, X., Ultrafast Viscous Water Flow through Nanostrand-Channelled Graphene Oxide Membranes. Nat Commun 2013, 4, 2979.

67. Liu, G.; Jin, W.; Xu, N., Graphene-Based Membranes. Chem. Soc. Rev. 2015, 44, 50165030.

68. Fine, D.; Grattoni, A.; Goodall, R.; Bansal, S. S.; Chiappini, C.; Hosali, S.; van de Ven, A. L.; Srinivasan, S.; Liu, X.; Godin, B.; Brousseau, L.; Yazdi, I. K.; Fernandez-Moure, J.; Tasciotti, E.; Wu, H.-J.; Hu, Y.; Klemm, S.; Ferrari, M., Silicon Micro- and Nanofabrication for Medicine. Adv. Healthcare Mater. 2013, 2, 632-666.

69. Schoch, R. B.; Han, J.; Renaud, P., Transport Phenomena in Nanofluidics. Reviews of Modern Physics 2008, 80, 839-883.

70. Vlassiouk, I.; Apel, P. Y.; Dmitriev, S. N.; Healy, K.; Siwy, Z. S., Versatile Ultrathin Nanoporous Silicon Nitride Membranes. Proceedings of the National Academy of Sciences of the United States of America 2009, 106, 21039-21044.

71. Yu, W. W.; White, I. M., Chromatographic Separation and Detection of Target Analytes from Complex Samples Using Inkjet Printed SERS Substrates. Analyst 2013, 138, 3679-3686. 
TOC Graphic

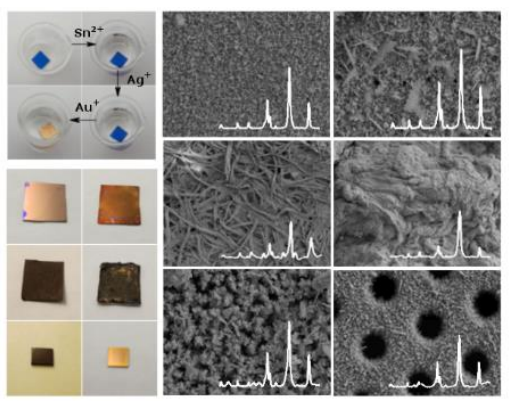

